\title{
HOMBRE Y NATURALEZA EN TOMAS DE AQUINO
}

Julio A. Castello Dubra*

SÍNTESIS - La doctrina de Tomás de Aquino respecto de la delimitación entre el ámbito de lo "natural" - entendiendo por tal la naturaleza "inferior", esto es, el ámbito de lo material, corporal y sensible - y el ámbito de lo humano puede ser reconstruida en tres momentos: a) una integración inicial del hombre al ámbito de lo natural - en particular por la doctrina de la unidad esencial de alma y cuerpo; b) una relativa emergencia o trascendencia del hombre respecto del lo material - en especial por la subsistencia del alma racional humana y la trascendencia de su fin último; c) una sintesis que marca la continuatio entre naturaleza y hombre, en la medida en que éste es concebido como "fin de toda la generación". La orientación teleológica de la naturaleza que tiende hacia el hombre, puede advertirse en la creciente perfección del diseño del orden de las formas naturales, en las condiciones fisicas propias de la corporalidad humana, y el en proceso generativo del embrión humano. En cada caso, al tender hacia el hombre, la naturaleza busca, en forma mediata, trascenderse a sí misma.

PALABRAS-CLAVE - Tomás de Aquino. Hombre y naturaleza. Alma y cuerpo.
ABSTRACT - The doctrine of Thomas Aquinas regarding the delimitation between the environment of the "natural" understanding for such the "inferior" nature, that is to say, the environment of the material, corporal and sensitive-and the environment of the human thing can be reconstructed in three moments: a) na initial integration of the man to the environment of the natural in particular for the doctrine of the essential unit of soul and body; b) a relative emergency or the man's transcendency regarding the the material specially for the subsistence of the human rational soul and the transcendency of its last end; c) a synthesis that marks the continuatio between nature and man, in the measure in that this is conceived as "end of the whole generation". The teleological orientation of the nature that spreads toward the man, can be noticed in the growing perfection of the design of the order in the natural ways, under the physical conditions characteristic of the human corporality, and in the generative process of the human embryo. In each case, when spreading toward the man, the nature looks for, in mediate form, to be transcended to itself. KEY WORDS - Thomas Aquinas. Man and nature. Soul and body.

La tradicional pregunta acerca de cuál es "el puesto del hombre en el cosmos" implica, en buena medida, una delimitación entre el ámbito de lo natural y de lo humano. Se trata de determinar cuál es la especificidad del hombre respecto del ámbito de la naturaleza y cuál es su relación con ella. Esto supone, a șu vez, hacer explícito qué es lo que entendemos por "naturaleza".

* Universidad de Buenos Aires.

\begin{tabular}{|l|l|l|l|l|l|}
\hline VERITAS & Porto Alegre & v. 44 & n. 3 & Setembro 1999 & p. 621-632 \\
\hline
\end{tabular}


Si queremos trasladar la cuestión a la perspectiva de Tomás de Aquino, habrá que revisar, por tanto, los diversos sentidos que tiene el término "naturaleza" y su adjetivo derivado "natural".' Inicialmente relacionado con la noción de nacimiento (cf. el participio futuro de nascor: naturus, -a, -um), se presenta como el equivalente latino del término técnico physis de Aristóteles, el "principio inmanente del movimiento y del reposo", en los dos sentidos en que lo interpreta Aristóteles: la materia y la forma. Si Tomás entiende que la esencia de las substancias compuestas estriba precisamente en la composición de materia y forma, es razonable que el término acabe convirtiéndose en un sinónimo más de essentia, pero con el matiz peculiar de señalar la función u operación propia en la que se manifiesta el ser esencial y actual de la cosa. ${ }^{2}$ Así decimos que es "natural" al cuerpo liviano el tender hacia arriba, a un lugar que precisamente por ello es calificado como "natural".

Tomado en este sentido, el término naturaleza mientras la esencia particular y propia de una cosa, se trata de la naturaleza de algo. Pero el término también puede aplicarse a delinear diversos ámbitos o dominios de la realidad. Y puede llegar a hacerlo en una forma tan amplia como para identificarse con la totalidad misma de lo real. En efecto, con "naturaleza" puede aludirse no sólo a cualquier creatura - sea substancia o accidente - sino incluso al agente mismo de la creación - Dios - pero en tanto natura naturans. Y así hablamos, por oposición al mero ens rationis, del ens naturae, o de lo que se da in natura, queriendo significar con ello lo que se da en la realidad. Finalmente, dentro de este sentido amplio de natura, cabe delimitar un sentido restringido que se refiere particularmente al ámbito de las cosas "desprovistas de razón", esto es, el mundo "físico", o aún más restrictivamente, el mundo "sublunar". Y en tal sentido se habla, en la acepción más cercana al uso actual, de la "naturaleza inferior" como el dominio de lo material, corporal y sensible, y se califica de "naturales" a los seres comprendidos en dicho ámbito y que revisten tales características.

Hechas estas precisiones, estamos en condiciones de preguntarnos cuál es la posición de Tomás de Aquino acerca de la delimitación entre el ámbito de lo natural - entendiendo por tal el último sentido señalado, la "naturaleza" en sentido restringido o "naturaleza inferior" - y el ámbito de lo propiamente humano 0 , en otras palabras, cuál es la relación - si de integración, confluencia, u oposición - entre los dominios de la realidad que solemos caracterizar bajo los títulos de "naturaleza" y "hombre".

Para caracterizar la respuesta de Tomás de Aquino a esta pregunta es preciso reconstruir los aspectos esenciales de la doctrina tomista acerca de la naturaleza del alma humana y de las relaciones entre alma y cuerpo. Dicha reconstrucción puede articularse, a los fines de su comprensión, en tres momentos. En primer término, Tomás subraya decisivamente la participación e integración inicial del hombre en el ámbito de lo natural. En efecto, la doctrina tomista acerca de la

Cf. Schütz, L., Thomas-Lexicon, Stuttgart, 1958, p. 509-10, 515-16.

Cf. De ente cap. 2. 
unión esencial de alma y cuerpo tiene la significación principal de recuperar la corporalidad como un componente inseparable de la esencia humana. El hombre no es un "alma que usa de un cuerpo", sino que alma y cuerpo, como que son forma y materia, constituyen un unum simpliciter, una única substancia. ${ }^{3}$ Ello contribuye a reafirmar el valor del cuerpo como un substrato natural constitutivo del hombre. La unión con el cuerpo es natural al alma humana y, por tanto, conveniente a ella; el estar unida al cuerpo resulta un beneficio para el alma y en modo alguno una "prisión". ${ }^{4}$ Pero al mismo tiempo, el alma no es el hombre sino sólo una "parte" del hombre. ${ }^{5}$ Por todo ello, el hombre puede ser calificado, abiertamente y sin reparos, como un "ser natural".

En un segundo momento, sin embargo, es igualmente evidente que el hombre emerge o se destaca del ámbito de la naturaleza inferior de la cual él participa, y ello por la especificidad de su alma, en virtud de la cual participa también del orden superior de la naturaleza - tomada ahora en su sentido más amplio constituido por el orden de las substancias intelectuales. El alma humana tiene una operación propia en la cual no participa el cuerpo, la intelección, y por ello constituye una forma "no totalmente inmersa en la materia" y como elevada por sobre ella, a tal punto que su vinculación con el cuerpo no le resta el carácter de un ser subsistente que permanece a la corrupción del cuerpo: ${ }^{7}$ en otras palabras, el alma humana es inmaterial e inmortal. Y aunque para desarrollar su actividad intelectiva necesita, ciertamente, de un material aportado por los sentidos, con su modo específico de intelección entra a formar parte ya del ámbito de las inteligencias, junto a las inteligencias separadas - ángeles - y la inteligencia suprema, que es Dios. Por esta posición intermedia en el orden de lo real, el alma humana resulta ser precisamente un "horizonte" o "confin" entre el ámbito de lo corporal y el de lo espiritual, ${ }^{8}$ y en la medida en que, a su manera, el hombre compendia en sí todas las perfecciones que se hallan en lo real, es considerado un "microcosmos" o "mundo en miniatura".

Pero el hombre emerge o se destaca del orden de la "naturaleza inferior" corporal y sensible - particularmente en cuanto que su fin es el fin último de toda la realidad. Todas las creaturas tienden hacia el fin último, tienden a asimilarse a Dios, pero lo consiguen de diversas maneras y en la medida de sus posibilidades: en cambio, el fin natural de toda substancia intelectual es contemplar a Dios. ${ }^{10} \mathrm{El}$ fin del alma humana es "trascender todo el orden de las creaturas y alcanzar el fin supremo que es Dios". "En verdad, en la consecución de su fin último, el hombre

\footnotetext{
Cf. SCG II 68; ST I q. 76 a. 1; De an. a. 1.

Cf. ST I q. 76 , a. 5 ; De an. a. 8.

Cf. ST I q. 75, a. 4; SCG II 57 §§ 5-6; De spir. creat. a. 2, ad $5^{\text {um }}$.

Cf. "Homo autem res naturalis est" (SCG IV 84, §5); "Animal enim et homo sunt quaedam sensibilia et naturalia" (cf. SCG II $57, \S 5$ ).

Cf. ST I q. 75, a. 2; SCG II 68, § 12; De an. a. 1.

Cf. ST I q. 98, a. 1; SCG II 68, § 6; $81 \S 12$; III 61, § 5; De an. a. 1; In Lib. De causis 2, 61-62, etc.

Cf. ST I q. 91, a. 1; In VIII Phys. 1. 4, n. 3; De pot. q. 5, a. 6, arg. 8; etc.

Cf. SCG III 25.

11 "Finis autem animae humanae et ultima perfectio eius est quod per cognitionem et amorem transcendat totum ordinem creaturarum et pertingat ad primum principium, quod Deus est." (SCG II 87 , § 6).
} 
no sólo trasciende la naturaleza inferior, sino hasta las posibilidades de su naturaleza - entendiendo ahora por tal su esencia - puesto que para el hombre la adquisición del fin último - la visión beatífica de Dios - es imposible de alcanzar por sus propias facultades naturales y requiere de la influencia sobrenatural de Dios.

Como puede verse, la delimitación tomista entre el orden de lo natural y el de lo humano se mantiene dentro de un cuidadoso equilibrio. El hombre participa substancialmente del ámbito de lo natural, a tal punto que ha de ser considerado inicialmente como uno más entre los seres naturales y sensibles; pero por otra parte, el hombre trasciende el orden natural, no sólo en cuanto participa del orden espiritual, sino en cuanto por dicha participación está destinado a una trascendencia total respecto de todo el orden de lo creado y participar así del privilegio de alcanzar el fin último de toda la realidad.

Sin embargo, como para que no queden dudas de la concepción armónica e integral que Tomás tiene de la naturaleza y del hombre, puede reconstruirse un tercer momento de esta delimitación, en el que Tomás señala la continuidad o enlace que va de la naturaleza inferior al hombre. Entre la naturaleza y el hombre no existe un hiato, o un "salto" que los oponga en una distancia insalvable, sino que la naturaleza misma tiende hacia el hombre, como que tiene en él su fin.

III

El orden de las formas naturales, constituido por las formas de los cuerpos primarios (fuego, agua, etc.) las formas de los cuerpos mixtos, y las formas correspondientes a los tres grados anímicos (almas vegetativa, sensitiva y, en último término, el alma racional humana, es pasible de una cierta consideración "dinámica" que está dada por las diversas referencias de cada uno de los niveles a los superiores: en el diseño jerárquico de creciente perfección es posible ver cierta tendencia o "aspiración" del conjunto de la escala y de cada uno de sus órdenes a alcanzar la forma más perfecta como el fin mismo de toda ella:

"Quanto igitur aliquis actus est posterior et magis perfectus, tanto principalius in ipsum appetitus materiae fertur. Unde oportet quod in ultimum et perfectissimum actum quem materia consequi potest, tendat appetitus materiae quo appetit formam, sicut in ultimum finem generationis. In actibus autem formarum gradus quidam inveniuntur. Nam materia prima est in potentia primo ad formam elementi. Sub forma vero elementi existens est in potentia ad formam mixti: propter quod elementa sunt materia mixti. Sub forma autem mixti considerata, est in potentia ad animam vegetabilem: nam talis corporis anima actus est. Itemque anima vegetabilis est in potentia ad sensitivam; sensitiva vero ad intellectivam. [...] Post hanc autem formam non invenitur in generabilibus et corruptibilibus posterior forma et dignior. Ultimus igitur finis generationis totius est anima humana, et in hac tendit materia sicut in ultimam formam. Sunt ergo elementa propter corpora mixta; haec vero propter viventia; in quibus plantae sunt propter animalia; animalia vero propter hominem. Homo igitur est finis totius generationis." ${ }^{12}$

${ }^{12}$ SCG III 22, §7. 
Según vemos, el orden debe considerarse como un despliegue de diversos grados de actualidad. Es por ello que el "apetito" inicial de la materia se extiende por sus diversos grados de potencialidad en busca del nivel más alto posible de actualidad, que reside, como ya sabemos, en el alma humana en tanto forma subsistente por sí y "emergente" de la materia. De hecho, la apetencia por la forma con la que se caracteriza a la materia está íntimamente relacionada con la noción de finalidad; y con el término relativo de perfección que ésta implica:

"Unaquaeque res naturaliter appetit perfectionem sui. Unde et materia dicitur appetere formam sicut imperfectum appetit suam perfectionem. ${ }^{13}$

"Non solum autem aliquid ens in actu per virtutem activam ordinatur in suum finem, sed etiam materia secundum quod est in potentia: nam forma est finis materiae. Nihil igitur est aliud materiam appetere formam, quam eam ordinari ad formam ut potentia ad actum. ${ }^{14}$

En consecuencia, el hombre aparece como el fin de toda la obra emprendida por la naturaleza en su búsqueda de la forma "más digna" posible dentro de los límites de los seres sometidos a la generación y la corrupción. El diseño de la escala revela así su sentido teleológico en la tendencia original de la materia a alcanzar la determinación en acto más perfecta posible dentro del marco en el que se despliega: el alma humana como forma ya "no totalmente inmersa" en la materia en razón de la relativa inmaterialidad de su modo de ser y su operación propios.

El significado de esta referencia progresiva de los niveles inferiores de lo natural "en vista" del hombre tiene su expresión más inmediata, en principio, en la provisión del sustento necesario en orden a la "conservación del ser". Puesto que por la misma cosa algo recibe la generación y tiene la conservación de su ser, ambos órdenes deben resultar necesariamente paralelos. Efectivamente, los cuerpos mixtos o compuestos, "se sustentan" de los elementos o cuerpos primarios - en tanto se componen de ellos; las plantas se nutren de los cuerpos mixtos, y los animales se alimentan de aquéllas - y entre los animales, los más perfectos y más fuertes se alimentan de los más débiles. Corroborando su puesto eminente, y su condición de fin de todas las demás creaturas, el hombre usa de todas ellas indistintamente para su provecho, ya sea para el alimento, el vestido y la protección, o la movilidad y el transporte..$^{15}$

Pero la referencia del conjunto de los seres naturales hacia el hombre no sólo comprende a los habitantes del "mundo sublunar", sometidos a la generación y la corrupción, sino que incluso se extiende, más allá de ese límite, a los astros y al

\footnotetext{
In I Met., l. 1, n. 2.

In I Phys. 1. 15, n. 138.

Tomás recoge así el antiguo locus de la aparente debilidad e indigencia de la condición inicial de indigencia humana, antes bien como un signo del dominio que se le ha reservado sobre las creaturas inferiores: "unde et a natura nudus est institutus, utpote potens ex aliis sibi vestitum praeparare; sicut etiam nullum sibi congruum nutrimentum natura praeparavit nisi lac, ut ex diversis rebus sibi cibum conquireret. [...] Unde et de homine in Psalmo dicitur [Sal. viii, 8.], ad Deum directo sermone: Omnia subiecisti sub pedibus eius. Et Aristoteles dicit, in I Politicorum [Cf. Pol. I 2, 1254b.] quod homo habet naturale dominium super omnia animalia." (SCG II 22, § 8).
} 
movimiento del cielo. A primera vista podría parecer inexplicable cómo seres más "nobles" y perfectos como lo son los astros en razón de su incorruptibilidad pueden estar en función de otros seres que pertenecen un nivel inferior. Pero ocurre que mientras algunos entes se procuran la semejanza divina en la búsqueda de la perpetuidad de la especie - en la reiteración de las generaciones, otros se asimilan a Dios, además de este modo, en cuanto son causas de otros seres subordinados a ellos. Así es que en verdad los astros manifiestan su superioridad y mayor nobleza al actuar como causa de la generación y la comupción en el mundo sublunar, en su intento por asimilarse a la generosa "difusividad" que caracteriza a la bondad divina. ${ }^{16}$ En última instancia, la obra de los astros no hace sino referirse mediatamente al hombre, en cuanto asegura, al organizar los ciclos generativos, la preservación de los órdenes inferiores de los cuales él se sustenta:

"Si igitur motio ipsius caeli ordinatur ad generationem, generatio autem tota ordinatur ad hominem sicut in ultimum finem huius generis: manifestum est quod finis motionis caeli ordinatur ad hominem sicut in ultimum finem in genere generabilium et mobilium." ${ }^{17}$

El hombre resulta así fin de toda la naturaleza sensible y móvil, incluyendo aquella que escapa a las limitaciones de la generación y la corrupción. En tal medida todo el devenir que domina en el ámbito de lo natural se orienta, en cierto sentido, hacia él.

Ahora bien, el orden de las formas naturales no sólo manifiesta un ascenso en el grado de perfección y actualidad de las formas, sino que implica además una jerarquía dentro de los niveles de la corporeidad misma. El despliegue de la naturaleza orientada teleológicamente hacia el hombre se comprueba también por el lugar de preferencia otorgado por Tomás a la corporeidad específica del hombre como fundamento propicio para el surgimiento de la intelectualidad. En efecto, el ascenso en la escala determina también distintos grados de perfección, desde los cuerpos más simples y rudimentarios, hasta los de composición más "refinada" y acabada. En este grado superior se halla el cuerpo humano, el cual se destacará por la adecuada composición de sus elementos, y las condiciones favorables de su disposición y contextura físicas.

Esta valoración de la corporeidad humana tiene su contexto en la demostración de la conveniencia de la unión del alma con su cuerpo, fundada en la necesidad de proveer a lo específico de la intelectualidad humana - su índole abstractiva - el "material" imprescindible para ejercer su operación propia. Así es como se explica que el alma humana haya sido dotada de un cuerpo que se revela finalmente como el más apto por sus condiciones naturales para el sentido, y especialmente para aquel de los sentidos que es considerado el primario, al par el que fundamento de todos los demás: el del tacto. ${ }^{18}$ Ahora bien, la aptitud

\footnotetext{
Cf. SCG III 22, § 5 .

Cf. SCG III 22, §9.

Cf. Arist. De an. II 2, 413b; III 12, 434a-b.
} 
sobresaliente del cuerpo humano para este sentido fundamental habrá de explicarse por los requerimientos de la función sensorial en los términos de la doctrina aristotélica de la sensación. En efecto, es preciso que cada órgano de los sentidos no posea en acto los contrarios de los sensibles que va a percibir, a fin de poder permanecer en potencia respecto de ellos. Pertenece a la índole misma de una naturaleza receptiva el estar desprovista de las determinaciones de lo que ha de recibir - v.gr., en la pupila no se hallan ni el blanco ni el negro, ni en general color alguno. Pero esto es imposible en el caso del órgano del tacto: siendo receptivo de determinaciones corporales como el calor y el frí, lo húmedo y lo seco, de estar enteramente desprovisto de todas ellas, no sería él mismo de naturaleza corporal. La única solución es que sus contrariedades sean "reducidas" a un perfecto medio, y así quedar en potencia respecto de ambas. La excelencia del cuerpo humano está dada precisamente por lo "delicado" de la proporción a la que se reducen sus contrariedades, por ser maxime reductum ad medium per aequalitatem complexionis. ${ }^{19}$

No está demás insistir en la importancia aquí concedida a un sentido como el tacto, cuya índole más "primitiva" y en mayor medida extensible al género animal en su conjunto no hacen sino confirmar una vez más la decisión de Tomás en favor del firme arraigo del hombre en lo natural, aún en un contexto en el que se considera a la corporeidad en función de su conveniencia para la operación intelectual. Sin duda, Tomás reconoce el carácter "primario" del tacto como el más rudimentario y menos refinado de los sentidos, en una jerarquía de progresiva inmaterialidad que coloca más bien a la vista en su cumbre. ${ }^{20}$ Con todo, no es menos cierto que el tacto sigue siendo "primario" ya no en el sentido limitante del término, sino en tanto fundante, no sólo de los restantes sentidos, sino incluso de la intelectualidad, más aún, del mejor o peor ejercicio de la misma. Siguiendo a Aristóteles, Tomás va llegar a tal punto en la valoración del tacto en cuanto condición de la inteligencia, que relacionará la presencia de una singular agudeza de este sentido con una mayor habilidad intelectual: Molles enim cames (qui sunt boni tactus) aptos mentes videmus. ${ }^{21}$

Pues bien, la adecuada complexio del cuerpo humano que favorece esta agudeza de la sensibilidad - y en tal medida, la posibilidad de la intelección también puede ser interpretada, por tanto, como la culminación de la progresiva perfección que el orden de la naturaleza inferior va desplegando en su búsqueda de las formas superiores. Todo el orden de las formas naturales parece tender así, a través de los distintos grados de la corporeidad, a la consecución del aquel cuerpo de mayor excelencia que obra como substrato del alma racional humana:

19 Cf. De an. a. 8.

Cf. ST I q. 78, a. 3. Del mismo modo se comenta el clásico texto aristotélico del comienzo de la Metafísica, acerca de la preferencia otorgada a la vista entre todos los sentidos: of. In I Met. 1. 1, n. 6.

De an. a. 8; cf. Arist. De an. II 9, 421a. 
"In quo apparet quod tota operatio inferioris naturae terminatur ad homines sicut ad perfectissimum. Videmus enim operationem naturae procedere gradatim a simplicibus elementis commiscendo ea, quousque perveniatur ad perfectissimum commixtionis modum, qui est in corpore humano. Hanc igitur oportet esse dispositionem corporis cui anima rationalis unitur, ut scilicet sit temperantissimae complexionis." ${ }^{22}$

Dentro de la excelencia que destaca a la corporalidad humana deben contarse además ciertas particularidades que conciemen a lo distintivo de la condición física del hombre en relación con el resto de los animales. Se trata de disposiciones orgánicas tales como el considerable volumen del cerebro en proporción al resto del cuerpo - que influye positivamente en las potencias sensitivas internas como la imaginación, la memoria, etc., o la colocación de la cabeza en la parte superior, acompañada de la posición erguida, que le otorga una incomparable libertad de acción. ${ }^{23}$ Todo ello contribuye, en última instancia, en beneficio del pleno y libre desarrollo de sus facultades intelectivas.

La corporalidad humana representa así el último eslabón en la tendencia de la naturaleza hacia el hombre, en tanto representa las condiciones físicas que únicamente hacen posible el surgimiento de la intelectualidad apoyada aún en el orden "inferior". El orden de las formas naturales va desplegando diversos grados de corporeidad hasta llegar al punto de mayor desarrollo en aquel ámbito propicio para el asentamiento de la primera manifestación del orden superior - el de las inteligencias. "La sabiduría de lo divino", que "conecta los fines de los seres inferiores con el comienzo de los superiores" ${ }^{24}$ no hace sino "preparar el terreno" para la aparición de la ínfima de las inteligencias proveyendo el substrato natural al cual deberá unirse esencialmente. Es ésta, pues, una de las formas en que el "apetito" de la materia por el ser en acto más perfecto tiende hacia él en un dinamismo que culmina en el hombre como fin del orden de la generación.

Por último, cabe señalar que este dinamismo con el que la naturaleza tiende hacia el hombre como a su fin se advierte con claridad en el escenario privilegiado de la generación del embrión humano, donde la sucesiva aparición de su vida vegetativa y sensitiva podrá interpretarse como una prefiguración de la recepción de la forma definitiva, que será el alma racional e inmortal.

El desarrollo de esta cuestión adquiere una singular relevancia por la necesidad de articular dos fuentes diversas: de un lado, la recepción de la doctrina aristotélica de la generación espermática en los animales evolucionados y, con ello, la necesidad de atribuir al embrión humano de una previa vida vegetativa, o incluso sensitiva, antes de la aparición definitiva del alma racional; del otro, el reconocimiento del origen sobrenatural del alma inmortal humana, creada personal e inmediatamente por Dios. El tratamiento más desarrollado que Tomás hace del tema se halla en la Summa contra Gentiles, y fundamentalmente, en el extenso artículo noveno de la tercera de las Quaestiones disputatae de potentia - "acerca de si el alma racional viene al ser por creación o se transmite por el semen". Tras hacer una prolija recopilación de las principales opiniones vertidas sobre la cuestión y el examen de sus posibles dificultades, Tomás de Aquino se decide por

22 De an. a. 8.

23 Cf. ibid.

${ }^{24}$ Frase de Pseudo-Dionisio frecuentemente citada por Tomás: cf. De div. nom. vii, 3. 
la doctrina de la generación del "múltiple" o compleja del embrión. A diferencia de la generación de cuerpos simples como el aire, fuego, etc., en los que la aparición de la nueva forma sucede instantáneamente a la desaparición de la anterior, en la generación de seres de alta complejidad como el hombre el proceso generativo comprende en verdad una serie de múltiples generaciones y corrupciones. El semen contiene inicialmente no el alma, sino una virtus del alma, que se aloja en cierto calor o spinitus, cuya presencia se ve facilitada por la naturaleza espumosa del semen. La potencia generativa va desarrollando así distintas formas substanciales de diversa especie - primero el semen, luego la sangre, etc. - con el objeto de "disponer" la materia para la recepción de la forma substancial definitiva. A ella se llega a través de un proceso en el que se suceden generaciones de almas de diverso grado y sus respectivas corrupciones, hasta legar, en el "último intento", a la forma más "evolucionada", que comprende en sí los grados anteriores:

"Sic ergo per virtutem formativam, quae a principio est in semine, abiecta forma spermatis, inducitur alia forma; qua abiecta iterum inducatur alia: et sic primo inducatur anima vegetabilis; deinde ea abiecta, inducatur anima sensibilis et vegetabilis simul; qua abiecta inducatur non per virtutem praedictam sed a creante, anima quae simul est rationalis sensibilis et vegetabilis." ${ }^{25}$

La elección de esta solución reconoce explícitamente la intención originaria de resguardar las dos premisas básicas que subyacen a toda la argumentación: la "nueva" doctrina aristotélica acerca de la vida vegetativa y sensitiva del embrión, conciliada con la doctrina de la unidad del alma, y la trascendencia del origen del alma inmortal humana, procedente de la intervención creadora de Dios:

"Et sic dicendum est secundum hanc opinionem quod embryo antequam habeat animam rationalem, vivit et habet animam, qua abiecta, inducitur anima rationalis. Et sic non sequitur duas animas esse in eodem corpore, nec animam rationalem traduci cum semine."26

Es así como el desarrollo de la vida embrional muestra en uno de sus momentos privilegiados el esfuerzo de la naturaleza en su tendencia hacia el surgimiento de lo humano. La sucesión de generaciones y comupciones previas a la aparición de la definitiva forma sustancial representa, en cierto modo, en un proceso genético y temporal, la misma referencia teleológica de los distintos grados de la escala de las formas naturales hacia su cumbre en el alma racional humana. El proceso de generación múltiple da lugar a verdaderas formas "intermedias" entre la materia y la última forma substancial, en uno de los únicos y excepcionales sentidos en que ello es admitido sin contradecir la doctrina de la unidad de la forma substancial. Sólo que las formas intermedias no llegan a constituir una especie completa, sino que ofician como disposiciones "provisorias" esencialmente orientadas a la consecución de la especie definitiva, en la cual únicamente hallan su sentido: 
"Nec est inconveniens si aliquid intermediorum generatur et statim postmodum interrumpitur: quia intermediam non habet speciem completam, sed sunt ut in via ad speciem; et ideo non generatur ut permaneant, sed ut per ea ad ultimum generatum perveniatur." ${ }^{27}$

Es precisamente en razón de la posición cumbre que detenta el alma humana dentro del orden de las formas naturales que la naturaleza debe proceder a través de distintos y sucesivos pasos en el proceso que desarrolla genéticamente su tendencia hacia el hombre:

"Quanto igitur aliqua forma est nobilior et magis distans a forma elementi, tanto oportet esse plures formas intermedias, quibus gradatim ad formam ultimam veniatur, et per consequens plures generationes medias." ${ }^{20}$

Sin embargo, podría considerarse problemático el que en este proceso las dos primeras generaciones relativas a las almas vegetativa y sensitiva, lo mismo que la del cuerpo en general, procedan seminalmente, al propio estilo de sus correspondientes causas naturales, mientras que el alma racional surge de la irrupción de un agente sobrenatural como lo es Dios. O bien, en términos de una de las difficultates enumeradas en la respectiva quaestio, parecería imposible que la acción de dos agentes distintos culmine, la de uno en la materia, y la del otro en la forma. De ser así, de la forma y de la materia de tal modo generadas no resultaría algo uno simpliciter como ya sabemos es el compuesto sustancial humano. ${ }^{29}$

La respuesta a esta ratio adversa nos muestra una vez más otro aspecto en el que la naturaleza se conduce hacia el surgimiento de lo humano. Por cierto que la acción separada de dos agentes diversos no puede concluir en la unidad esencial de un compuesto de materia y forma, "siempre que no se trate de dos agentes coordinados" - agrega Tomás - en cuyo caso un agente actúa como instrumento del otro. En tal caso, es natural que la acción del agente principal se extienda al término hacia el cual el agente instrumental no puede por su sola naturaleza llegar. Ahora bien, es sabido que la naturaleza obra en cierto modo como causa segunda instrumental respecto de la acción de la causa primera divina (instrumentum quoddam divinae virtutis). En consecuencia no hay inconveniente alguno en afirmar que la acción de la naturaleza se limita a "disponer la materia" - generación del cuerpo - para que se halle en condiciones aptas de recibir la respectiva forma - infusión sobrenatural del alma racional. ${ }^{30}$

z7 SCG II 89, § 10. Los únicos dos casos en que puede hablarse de "mediación" en la unión entre alma y cuerpo son en el presente caso de la generación, y en el de la relación de motor a movido (cf. SCG II 71, § 3). SCG II 89 , § 11 .

Cf. De pot. q. 3, a. 9, arg. 21; SCG II 88, § 4 [arg. 3].

30 De pot. q. 3, a. 9, ad $21^{\mathrm{um}}$. Por lo demás, hay que decir que la infusión del alma en el cuerpo es simultánea con su creación, y se identifica con ella. El rechazo de la "preexistencia" de las almas a partir de una creación anterior a su corporalización muestra un aspecto más del enfrentamiento con la tradición neoplatonizante. Cf. ST I q. 90, a. 4; q. 118, a. 3; SCG If 83-84; De pot. q. 3, a. 10. 
Así como habiamos interpretado anteriormente la excelencia de la corporalidad humana como la "preparación" del terreno propicio para el surgimiento de la intelectualidad en algunas condiciones físicas que lo hacen posible, del mismo modo vemos ahora cómo en su acción generadora la naturaleza obra como instrumento de la acción divina, llevando a cabo la efectiva tarea de disponer la materia para la aparición de una forma que está más allá de sus solos recursos. El origen sobrenatural del alma racional humana, a la vez que supone la orientación de la acción de la naturaleza hacia el hombre, confirma la trascendencia de éste respecto de los límites del marco de la mera naturaleza "inferior", la cual que se muestra incapaz de alcanzar por sí sola la culminación de la especificidad de lo humano:

"[...] impossibile est actionem corporeae virtutis ad hoc elevari quod virtutem penitus spiritualem et incorpoream causare possit. ${ }^{\text {"31 }}$

La trascendencia del alma, que convoca una fuente peculiar para su generación, responde a los términos ya conocidos de la "emergencia" sobre la materia en razón de su subsistencia, fundada en la inmaterialidad de su operación propia - la intelección - que no se realiza mediante órgano corporal alguno. Un modo de generación comprometido con factores corporales y materiales, como lo es la generación natural espermática, resulta incompatible con sus singulares propiedades. Por tanto, habrá que atribuir al alma un fieri distinto del que procede ex materia, y que no puede ser sino ex nihilo, esto es, por creación. De lo contrario, su situación se asimilaría a la de las restantes formas no subsistentes:

"Ponere autem quod per generationem corporis fiat [sc. anima rationalis] est ponere ipsam non esse subsistentem, et per consequens cum corpori corrumpi. ${ }^{32}$

La inmaterialidad del alma representa un grado inalcanzable para las solas posibilidades de las causas que dominan el orden de las formas naturales, pese a que el diseño teleológico que anima la estructura de tal orden se orienta esencialmente a ella, tal como lo revela su transposición efectiva al orden de la ontogénesis. La naturaleza se encamina de tal modo hacia un fin que requiere ya de la intervención de un agente que exceda el marco de sus limitaciones. Se requerirá para ello, ya no de un mero agente superior, sino en verdad del máximo agente posible, el único capaz de producir una forma de tal excelencia. ${ }^{33}$

Ibid.

La infusión del alma es, por tanto, de carácter sobrenatural, pero no milagrosa: se trata simplemente de la necesidad de apelar al único agente capaz de producir el efecto requerido - vista la imposibilidad de la naturaleza sola en este caso. Por lo demás, la creación del alma en particular no es un milagro, como tampoco lo es la creación del mundo en general. Cf. In II Sent. d. 18, q. 1, a. 3, ad $1^{\text {um }}$. 
Del breve itinerario recorrido podemos concluir que, tanto en el diseño del orden de las formas naturales, en la excelencia de la corporalidad humana, y en la acción instrumental de la causalidad natural en la génesis embrional, es posible advertir cómo el dinamismo intrínseco de la naturaleza tiende hacia el hombre como a su fin. Pero es importante observar que en cada uno de estos aspectos, esta tendencia de la naturaleza se encamina hacia lo humano en los términos precisos de su especificidad. Si ésta ha sido caracterizada por la tendencia a trascender los límites iniciales del marco natural - la emergencia y el "dominio" sobre la materia, y la trascendencia del fin último del hombre - la conclusión es que la orientación esencial de la naturaleza no busca sino trascenderse a sí misma.

En efecto, el "apetito" inicial de la materia a través de la escala de las formas naturales, en su búsqueda del acto más perfecto, tendía necesariamente al alma humana en su peculiar condición de forma "no totalmente inmersa en la materia", y con "dominio" e independencia de ella. Por su parte, es redundante decir que la aptitud de la corporalidad humana para el asentamiento de la intelectualidad, apunta, en igual sentido, hacia el desarrollo de la condición racional específica del hombre, su inteligencia abstractiva. Por último, la orientación teleológica de la génesis embrional señala un itinerario que persigue la realización de la forma más "evolucionada" en el alma racional humana, cuya inmaterialidad y subsistencia requieren ya un origen trascendente en Dios.

Ahora bien, puesto que el fin del alma humana es trascender no sólo el marco de la naturaleza inferior, sino todo el orden de la creación - e incluso la "propia" naturaleza, es decir, sus propias facultades naturales - puede decirse que la "preparación" con la que la naturaleza tiende hacia el surgimiento de lo humano implica también, en cierta medida, la orientación hacia un fin en el cual la propia naturaleza se trasciende a sí misma, y a través del cual se dirige hacia el fin absoluto que por sí misma no puede alcanzar. En definitiva, la naturaleza tiende hacia el hombre como el "mediador" a través del cual busca su asimilación a Dios:

"Sed quia optimo assimilatur aliquid per hoc quod simile fit meliore se, ideo omnis creatura corporalis tendit in assimilationem creaturae intellectualis quantum potest, quae altiori modo divinam bonitatem consequitur, et propter hoc etiam forma humana, scilicet anima rationalis, dicitur esse finis ultimus intentus a natura inferiori $[\ldots] .^{n 4}$

A la naturaleza se le atribuye la expresa "intención" de alcanzar al hombre, en su tendencia a asimilarse al fin último. La trascendencia de lo natural que pertenece al hombre no repercute así en un "distanciamiento" final que introduzca una suerte de ruptura o discontinuidad en la secuencia que va de la naturaleza hacia él. Por el contrario, la naturaleza persigue como fin precisamente esta parcial trascendencia de lo humano, como un modo de encaminarse a la trascendencia absoluta a la que no puede dejar de referirse todo ser creado, y en donde se halla, desde la perspectiva de Tomás de Aquino, el sentido último de toda la realidad.

In II Sent. d. 1, q. 2, a. 3. 